\title{
Robust estimation in flat fading channels under bounded channel uncertainties
}

\author{
Mehmet A. Donmez*, Huseyin A. Inan, Suleyman S. Kozat \\ Department of ECE, Koc University, Istanbul, Turkey
}

\section{A R T I C L E I N F O}

\section{Article history:}

Available online 31 May 2013

\section{Keywords:}

Channel equalization

Flat fading

Minimax

Minimin

Minimax regret

\begin{abstract}
A B S T R A C T
We investigate channel equalization problem for time-varying flat fading channels under bounded channel uncertainties. We analyze three robust methods to estimate an unknown signal transmitted through a time-varying flat fading channel. These methods are based on minimizing certain meansquare error criteria that incorporate the channel uncertainties into their problem formulations instead of directly using the inaccurate channel information that is available. We present closed-form solutions to the channel equalization problems for each method and for both zero mean and nonzero mean signals. We illustrate the performances of the equalization methods through simulations.
\end{abstract}

(c) 2013 Elsevier Inc. All rights reserved.

\section{Introduction}

In this paper, we study channel equalization problem for time-varying flat (frequency-nonselective) fading channels under bounded channel uncertainties [1-7]. In this widely studied framework, an unknown desired signal is transmitted through a discretetime time-varying channel and corrupted by additive noise where the mean and variance of the desired signal is assumed to be known. Although the underlying channel impulse response is not known exactly, an estimate and an uncertainty bound on it are given [4-6]. Here, we investigate three different channel equalization frameworks that are based on minimizing certain meansquare error criteria. These channel equalization frameworks incorporate the channel uncertainties into their problem formulations to provide robust solutions to the channel equalization problem instead of directly using the inaccurate channel information that is available to equalize the channel. Based on these frameworks, we analyze three robust methods to equalize time-varying flat fading channels. The first approach we investigate is the affine minimax equalization method $[5,8,9]$, which minimizes the estimation error for the worst case channel perturbation. The second approach we study is the affine minimin equalization method $[6,10]$, which minimizes the estimation error for the most favorable perturbation. The third approach is the affine minimax regret equalization method $[4,5,11,7]$, which minimizes a certain "regret" as defined in Section 2 and further detailed in Section 3. We provide closedform solutions to the affine minimax equalization, the minimin equalization and the minimax regret equalization problems for

\footnotetext{
* Corresponding author.

E-mail addresses: medonmez@ku.edu.tr (M.A. Donmez), hinan@ku.edu.tr (H.A. Inan), skozat@ku.edu.tr (S.S. Kozat).
}

both zero mean and nonzero mean signals. Note that the nonzero mean signals frequently appear in iterative equalization applications $[11,12]$ and equalization with these signals under channel uncertainties is particularly important and challenging.

When there are uncertainties in the channel coefficients, one of the prevalent approaches to find a robust solution to the equalization problem is the minimax equalization method $[9,5,8]$. In this approach, affine equalizer coefficients are chosen to minimize the MSE with respect to the worst possible channel in the uncertainty bounds. We emphasize that although the minimax equalization framework has been introduced in the context of statistical signal processing literature $[9,5,8]$, our analysis significantly differs since we provide a closed-form solution to the minimax equalization problem for time-varying flat fading channels. In [5], the uncertainty is in the noise covariance matrix and the channel coefficients are assumed to be perfectly known. Furthermore, note that in [8], the minimax estimator is formulated as a solution to a semidefinite programming (SDP) problem, unlike in here. In this paper, the uncertainty is in the channel impulse response and we provide an explicit solution to the minimax channel equalization problem.

Although the minimax equalization method is able to minimize the estimation error for the worst case channel perturbation, however, it usually provides unsatisfactory results on the average [6]. An alternative approach to the channel equalization problem is the minimin equalization method $[6,10]$. In this approach, equalizer parameters are selected to minimize the MSE with respect to the most favorable channel over the set of allowed perturbations. Although the minimin approach has been studied in the literature $[6,10]$, however, we emphasize that to the best of our knowledge, this is the first closed-form solution to the minimin channel equalization problem for time-varying flat fading channels. 
The minimin approach is highly optimistic, which could yield unsatisfactory results, when the difference between the underlying channel impulse response and the most favorable channel impulse response is relatively high [6]. In order to preserve robustness and counterbalance the conservative nature of the minimax approach, the minimax regret approaches have been introduced in the signal processing literature $[4,13,7]$. In this approach, a relative performance measure, i.e., "regret", is defined as the difference between the MSE of an affine equalizer and the MSE of the affine minimum MSE (MMSE) equalizer [7]. The minimax regret channel equalizer seeks an equalizer that minimizes this regret with respect to the worst possible channel in the uncertainty region. Although this approach has been investigated before, the minimax regret estimator is formulated as a solution to an SDP problem [4], unlike here. In this paper, we explicitly provide the equalizer coefficients and the estimate of the desired signal.

Our main contributions are as follows. We first formulate the affine equalization problem for time-varying flat fading channels under bounded channel uncertainties. We then investigate three robust approaches; affine minimax equalization, affine minimin equalization, and affine minimax regret equalization for both zero mean and nonzero mean signals. The equalizer coefficients, and hence, the MSE of each methods have been explicitly provided, unlike in $[4,5,8,6,7]$.

The paper is organized as follows. In Section 2, the basic transmission system is described, along with the notation used in this paper. We present the affine equalization approaches in Section 3. First, we study the affine minimax equalization tuned to the worst possible channel filter. We then investigate the minimin approach and the minimax regret approach, and provide the explicit solutions to the corresponding optimization problems. In addition, we present and compare the MSE performances of all robust affine equalization methods in Section 4. Finally, we conclude the paper with certain remarks in Section 5.

\section{System description}

In this section, we provide the basic description of the system studied in this paper. Here, the signal $x_{t}$ is transmitted through a discrete-time time-varying channel with a channel coefficient $h_{t}$, where $x_{t}$ is unknown and random with known mean $\overline{x_{t}} \triangleq E\left[x_{t}\right]$ and variance $\sigma_{x}^{2} \triangleq E\left[\left(x_{t}-\overline{x_{t}}\right)^{2}\right]$. The received signal $y_{t}$ is given by

$y_{t}=x_{t} h_{t}+n_{t}$,

where the observation noise $n_{t}$ is independent and identically distributed (i.i.d.) with zero mean and variance $\sigma_{n}^{2}$ and independent from $x_{t}$. We consider a time-varying flat fading channel, where the bandwidth of the transmitted signal $x_{t}$ is much smaller than the channel bandwidth so that the multipath channel simply scales the transmitted signal $[14,15]$. However, instead of the true channel coefficient, an estimate of $h_{t}$ is provided as $\tilde{h}_{t}$, where $\delta h_{t} \triangleq \tilde{h}_{t}-h_{t}$ is the uncertainty in the channel coefficient and is modeled by $\left|h_{t}-\tilde{h}_{t}\right|=\left|\delta h_{t}\right| \leqslant \epsilon, \epsilon>0, \epsilon<\infty$, where $\epsilon$ or a bound on $\epsilon$ is known.

We then use the received signal $y_{t}$ to estimate the transmitted signal $x_{t}$ as shown in Fig. 1. The estimate of the desired signal is given by

$$
\begin{aligned}
\hat{x}_{t} & =w_{t} y_{t}+l_{t} \\
& =w_{t}\left(x_{t} h_{t}+n_{t}\right)+l_{t},
\end{aligned}
$$

where $w_{t}$ is the equalizer coefficient. We note that in (2), the equalizer is "affine" where there is a bias term $l_{t}$ since the transmitted signal $x_{t}$, and consequently the received signal $y_{t}$, are not necessarily zero mean and the mean sequence $\bar{y}_{t} \triangleq E\left[y_{t}\right]$ is not known due to uncertainty in the channel.

Even under the channel uncertainties, the equalizer coefficient $w_{t}$ and the bias term $l_{t}$ can be simply optimized to minimize the MSE for the channel that is tuned to the estimate $\tilde{h}_{t}$, which is also known as the MMSE estimator [16]. The corresponding equalizer coefficient and the bias term are given by $[17,11]$

$\left\{w_{0, t}, l_{0, t}\right\}=\arg \min _{w, l} E\left[\left(x_{t}-w\left(\tilde{h}_{t} x_{t}+n_{t}\right)-l\right)^{2}\right]$.

However, the estimate

$\hat{x}_{0, t} \triangleq w_{0, t} y_{t}+l_{0, t}$

may not perform well when the error in the estimate of the channel coefficient is relatively high $[18,4,5]$. One alternative approach to find a robust solution to this problem is to minimize a worst case MSE, which is known as the minimax criterion, as

$$
\begin{aligned}
& \left\{w_{1, t}, l_{1, t}\right\} \\
& \quad=\arg \min _{w, l} \max _{\left|\delta h_{t}\right| \leqslant \epsilon} E\left[\left(x_{t}-w\left(\left(\tilde{h}_{t}+\delta h_{t}\right) x_{t}+n_{t}\right)-l\right)^{2}\right],
\end{aligned}
$$

where $w_{1, t}$ and $l_{1, t}$ minimize the worst case error in the uncertainty region $[8,16]$. However, this approach may yield highly conservative results, since the estimate

$\hat{x}_{1, t} \triangleq w_{1, t} y_{t}+l_{1, t}$

is formed by using the equalizer coefficient $w_{1, t}$ and the bias term $l_{1, t}$ that minimize the worst case error, i.e., the error under the worst possible channel coefficient $[6,4,5]$. Instead of this conservative approach, another useful method to estimate the desired signal is the minimin approach, where the equalizer coefficient and the bias term are given by

$$
\begin{aligned}
& \left\{w_{2, t}, l_{2, t}\right\} \\
& \quad=\arg \min _{w, l} \min _{\left|\delta h_{t}\right| \leqslant \epsilon} E\left[\left(x_{t}-w\left(\left(\tilde{h}_{t}+\delta h_{t}\right) x_{t}+n_{t}\right)-l\right)^{2}\right],
\end{aligned}
$$

where $w_{2, t}$ and $l_{2, t}$ minimize the MSE in the most favorable case, i.e., the MSE under the best possible channel coefficient [6]. The estimate of the transmitted signal $x_{t}$ is given by

$\hat{x}_{2, t} \triangleq w_{2, t} y_{t}+l_{2, t}$

A major drawback of the minimin approach is that it is a highly optimistic technique, which could yield unsatisfactory results, when the difference between the actual and the best channel coefficients is relatively high [6].

In order to reduce the conservative characteristic of the minimax approach as well as to maintain robustness, the minimax regret approach is introduced, which provides a trade-off between

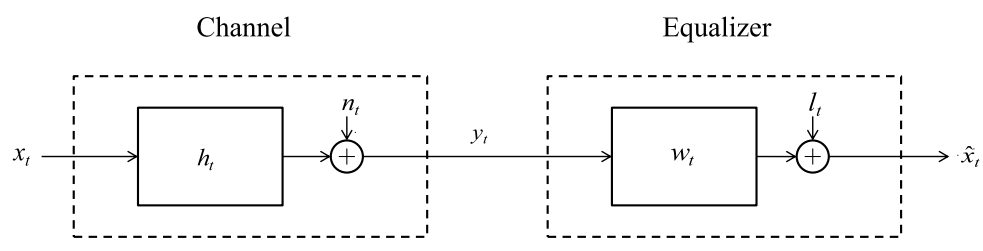

Fig. 1. A basic affine equalizer framework. 
performance and robustness $[4,11,7]$. In this approach, the equalizer coefficient and the bias term are chosen in order to minimize the worst-case "regret", where the regret for not using the MMSE is defined as the difference between the MSE of the estimator and the MSE of the MMSE, i.e.,

$$
\begin{aligned}
\left\{w_{3, t}, l_{t, 3}\right\}= & \arg \min _{w, l} \max _{\left|\delta h_{t}\right| \leqslant \epsilon}\left\{E\left[\left(x_{t}-w\left(\left(\tilde{h}_{t}+\delta h_{t}\right) x_{t}+n_{t}\right)-l\right)^{2}\right]\right. \\
& \left.-\min _{w, l} E\left[\left(x_{t}-w\left(\left(\tilde{h}_{t}+\delta h_{t}\right) x_{t}+n_{t}\right)-l\right)^{2}\right]\right\} .
\end{aligned}
$$

The corresponding estimate of the desired signal $x_{t}$ is given by

$\hat{x}_{3, t} \triangleq w_{3, t} y_{t}+l_{3, t}$

In the next section, we investigate and provide closed-form solutions for the three equalization formulations:

- affine minimax equalization framework,

- affine minimin equalization framework,

- affine minimax regret equalization framework.

We first solve the corresponding optimization problems and obtain the estimates of the desired signal. We next compare their meansquare error performances in Section 4.

\section{Equalization frameworks}

\subsection{Affine MMSE equalization}

In this section, we present the affine MMSE equalization framework for completeness $[11,16]$. Since the channel coefficient $h_{t}$ is not accurately known but estimated by $\tilde{h}_{t}$, a linear equalizer that is matched to the estimate $\tilde{h}_{t}$ and minimizes the MSE can be used to estimate the transmitted signal $x_{t}$. The corresponding equalizer coefficient $w_{0, t}$ and the bias term $l_{0, t}$ are given by (3).

We define $H(w, l)=E\left[\left(x_{t}-w\left(\tilde{h}_{t} x_{t}+n_{t}\right)-l\right)^{2}\right]$. Note that $H(w, l)$ is a quadratic function of the variables $w$ and $l$ where the coefficients of the terms $w^{2}$ and $l^{2}$ are positive. Hence, $H(w, l)$ is a convex function of $w$ and $l$. It follows that it has a global minimizer $\left(w^{*}, l^{*}\right)$, where $w^{*}$ and $l^{*}$ satisfy

$\left.\frac{\partial H}{\partial w}\right|_{w=w^{*}}=0,\left.\quad \frac{\partial H}{\partial l}\right|_{l=l^{*}}=0$.

Solving (7), we get

$w_{0, t}=\frac{\tilde{h}_{t} \sigma_{x}^{2}}{\tilde{h}_{t}^{2} \sigma_{x}^{2}+\sigma_{n}^{2}}, \quad l_{0, t}=\frac{\overline{x_{t}} \sigma_{n}^{2}}{\tilde{h}_{t}^{2} \sigma_{x}^{2}+\sigma_{n}^{2}}$.

\subsection{Affine equalization using a minimax framework}

In this section, we investigate a robust estimation framework based on a minimax criteria $[16,19,10]$. We find the equalizer coefficient $w_{1, t}$ and the bias term $l_{1, t}$ that solve the optimization problem (4).

In (4), we seek to find an equalizer coefficient $w_{1, t}$ and a bias term $l_{1, t}$ that perform best in the worst possible scenario. This framework can be perceived as a two-player game problem, where one player tries to pick $w_{1, t}$ and $l_{1, t}$ pair that minimize the MSE for a given channel uncertainty while the opponent pick $\delta h_{t}$ to maximize MSE for this pair. In this sense, this problem is constrained since there is a limit on how large the channel uncertainty $\delta h_{t}$ can be, i.e., $\left|\delta h_{t}\right| \leqslant \epsilon$ where $\epsilon$ or a bound on $\epsilon$ is known.

In the following theorem we present a closed-form solution to the optimization problem (4).
Theorem 1. Let $x_{t}, y_{t}$ and $n_{t}$ represent the transmitted, received and noise signals such that $y_{t}=h_{t} x_{t}+n_{t}$, where $h_{t}$ is the unknown channel coefficient and $n_{t}$ is i.i.d. zero mean with variance $\sigma_{n}^{2}$. At each time $t$, given an estimate $\tilde{h}_{t}$ of $h_{t}$ satisfying $\left|h_{t}-\tilde{h}_{t}\right| \leqslant \epsilon$, the solution to the optimization problem (4) is given by

$w_{1, t}= \begin{cases}\frac{\left(\tilde{h}_{t}-\epsilon\right) \sigma_{x}^{2}}{\left(\tilde{h}_{t}-\epsilon\right)^{2} \sigma_{x}^{2}+\sigma_{n}^{2}}: & \tilde{h}_{t} \epsilon \sigma_{x}^{2}<\epsilon^{2} \sigma_{x}^{2}+\sigma_{n}^{2}, \\ \frac{\sigma_{x}^{2}}{x_{t}^{2} \tilde{h}_{t}}: & \tilde{h}_{t} \epsilon \sigma_{x}^{2} \geqslant \epsilon^{2} \sigma_{x}^{2}+\sigma_{n}^{2}\end{cases}$

and

$l_{1, t}= \begin{cases}\frac{\overline{x_{t}} \sigma_{n}^{2}}{\left(\tilde{h}_{t}-\epsilon\right)^{2} \sigma_{x}^{2}+\sigma_{n}^{2}}: & \tilde{h}_{t} \epsilon \sigma_{x}^{2}<\epsilon^{2} \sigma_{x}^{2}+\sigma_{n}^{2}, \\ \overline{x_{t}}: & \tilde{h}_{t} \epsilon \sigma_{x}^{2} \geqslant \epsilon^{2} \sigma_{x}^{2}+\sigma_{n}^{2},\end{cases}$

where $\overline{x_{t}} \triangleq E\left[x_{t}\right]$ and $\sigma_{x}^{2} \triangleq E\left[\left(x_{t}-\overline{x_{t}}\right)^{2}\right]$ are the mean and variance of the transmitted signal $x_{t}$, respectively.

Proof. Here, we find the equalizer coefficient $w_{1, t}$ and the bias term $l_{1, t}$ that solve the optimization problem in (4). To accomplish this, we first solve the inner maximization problem and find the maximizer channel uncertainty $\delta h_{t}^{*}$. We then substitute $\delta h_{t}^{*}$ in (4) and solve the outer minimization problem to find $w_{1, t}$ and $l_{1, t}$.

We solve the inner maximization problem as follows. We observe that the cost function in (4) can be written as

$$
\begin{aligned}
& E\left[\left(x_{t}-w\left(\left(\tilde{h}_{t}+\delta h_{t}\right) x_{t}+n_{t}\right)-l\right)^{2}\right] \\
& \quad=w^{2} \Delta h_{t}^{2} \overline{x_{t}^{2}}+2 w \Delta h_{t}\left(l \overline{x_{t}}-\overline{x_{t}^{2}}\right)+\mathrm{C}_{1},
\end{aligned}
$$

where $\overline{x_{t}^{2}} \triangleq E\left[x_{t}^{2}\right], \Delta h_{t} \triangleq \tilde{h}_{t}+\delta h_{t}$ and $C_{1}=\overline{x_{t}^{2}}+w^{2} \sigma_{n}^{2}+l^{2}-2 l \overline{x_{t}}$ does not depend on $\delta h_{t}$. If we define $a=\overline{x_{t}^{2}}>0, b=l \overline{x_{t}}-\overline{x_{t}^{2}}, u=$ $w \Delta h_{t}$ and $C_{2}=C_{1}-\frac{b^{2}}{a}$, then (8) can be written as

$E\left[\left(x_{t}-w\left(\left(\tilde{h}_{t}+\delta h_{t}\right) x_{t}+n_{t}\right)-l\right)^{2}\right]=a\left(u+\frac{b}{a}\right)^{2}+C_{2}$

where $C_{2}$ is independent of $\delta h_{t}$. Hence the inner maximization problem in (4) can be written as

$$
\begin{aligned}
\delta h_{t}^{*} & =\arg \max _{\left|\delta h_{t}\right| \leqslant \epsilon} E\left[\left(x_{t}-w\left(\left(\tilde{h}_{t}+\delta h_{t}\right) x_{t}+n_{t}\right)-l\right)^{2}\right] \\
& =\arg \max _{\left|\delta h_{t}\right| \leqslant \epsilon} a\left(u+\frac{b}{a}\right)^{2} \\
& =\arg \max _{\left|\delta h_{t}\right| \leqslant \epsilon}\left|u+\frac{b}{a}\right|=\arg \max _{\left|\delta h_{t}\right| \leqslant \epsilon}\left|w \delta h_{t}+\frac{l \overline{x_{t}}-\overline{x_{t}^{2}}}{\overline{x_{t}^{2}}}\right| \\
& =\arg \max _{\left|\delta h_{t}\right| \leqslant \epsilon}|w|\left|\delta h_{t}+\frac{l \overline{x_{t}}-\overline{x_{t}^{2}}}{w \overline{x_{t}^{2}}}\right| .
\end{aligned}
$$

If we apply the triangular inequality to the second term in (9), then we get the following upper bound:

$$
\begin{aligned}
|w|\left|\Delta h_{t}+\frac{l \overline{x_{t}}-\overline{x_{t}^{2}}}{w \overline{x_{t}^{2}}}\right| & \leqslant|w|\left[\left|\delta h_{t}\right|+\left|\tilde{h}_{t}+\frac{l \overline{x_{t}}-\overline{x_{t}^{2}}}{w \overline{x_{t}^{2}}}\right|\right] \\
& \leqslant|w|\left[\epsilon+\left|\tilde{h}_{t}+\frac{l \overline{x_{t}}-\overline{x_{t}^{2}}}{w \overline{x_{t}^{2}}}\right|\right],
\end{aligned}
$$

where the upper bound is achieved at $\delta h_{t}^{*}=\epsilon \operatorname{sgn}\left(\tilde{h}_{t}+\frac{l \overline{x_{t}}-\overline{x_{t}^{2}}}{w \overline{x_{t}^{2}}}\right)$, where $\operatorname{sgn}(z)=1$ if $z \geqslant 0$ and $\operatorname{sgn}(z)=-1$ if $z<0$. Hence it follows that 


$$
\begin{aligned}
\delta h_{t}^{*} & =\arg \max _{\left|\delta h_{t}\right| \leqslant \epsilon} E\left[\left(x_{t}-w\left(\left(\tilde{h}_{t}+\delta h_{t}\right) x_{t}+n_{t}\right)-l\right)^{2}\right] \\
& = \begin{cases}\epsilon: & \tilde{h}_{t}+\frac{l \overline{x_{t}}-\overline{x_{t}^{2}}}{w \overline{x_{t}^{2}}} \geqslant 0, \\
-\epsilon: & \tilde{h}_{t}+\frac{l \overline{x_{t}}-\overline{x_{t}^{2}}}{w \overline{x_{t}^{2}}} \leqslant 0 .\end{cases}
\end{aligned}
$$

Note that if $\tilde{h}_{t}+\frac{l \overline{x_{t}}-\overline{x_{t}^{2}}}{w \overline{x_{t}^{2}}}=0$, then $\delta h_{t}^{*}=\epsilon$ and $\delta h_{t}^{*}=-\epsilon$ yields the same result.

We next solve the outer minimization problem as follows. We first note that the minimum in (4) is taken over all $w \in \mathbb{R}$ and $l \in \mathbb{R}$. If we write $\boldsymbol{u}=[w, l]^{T} \in \mathbb{R}^{2}$ in a vector form, define $\mathcal{U}=$ $\left\{\boldsymbol{u}=[w, l]^{T} \in \mathbb{R}^{2} \mid \tilde{h}_{t}+\frac{l \overline{x_{t}}-\overline{x_{t}^{2}}}{\overline{w x_{t}^{2}}} \geqslant 0\right\}$ and $\mathcal{V} \triangleq\left\{\boldsymbol{u}=[w, l]^{T} \in \mathbb{R}^{2} \mid\right.$ $\left.\tilde{h}_{t}+\frac{\overline{x_{t}}-\overline{x_{t}^{2}}}{w \overline{x_{t}^{2}}} \leqslant 0\right\}$, then it follows that $\mathcal{U} \cup \mathcal{V}=\mathbb{R}^{2}$. Hence, the cost function in the outer minimization problem in (4) is given by

$$
\begin{aligned}
& \max _{\left|\delta h_{t}\right| \leqslant \epsilon} E\left[\left(x_{t}-w\left(\left(\tilde{h}_{t}+\delta h_{t}\right) x_{t}+n_{t}\right)-l\right)^{2}\right] \\
& = \begin{cases}E\left[\left(x_{t}-w\left(\left(\tilde{h}_{t}+\epsilon\right) x_{t}+n_{t}\right)-l\right)^{2}\right]: & {[w, l]^{T} \in \mathcal{U},} \\
E\left[\left(x_{t}-w\left(\left(\tilde{h}_{t}-\epsilon\right) x_{t}+n_{t}\right)-l\right)^{2}\right]: & {[w, l]^{T} \in \mathcal{V} .}\end{cases}
\end{aligned}
$$

We first substitute $\delta h_{t}=\epsilon$ and find the corresponding $\{w, l\}$ pair that minimizes the objective function in (4) to check whether $[w, l] \in \mathcal{U}$. We next substitute $\delta h_{t}=-\epsilon$ and find the corresponding $\{w, l\}$ to check whether $[w, l] \in \mathcal{V}$. Based on these criteria, we obtain the corresponding equalizer coefficient and the bias term pair $\left\{w_{1, t}, l_{1, t}\right\}$.

We first substitute $\delta h_{t}=\epsilon$ in the objective function of (4) to get the following minimization problem:

$$
\begin{aligned}
\left\{w^{*}, l^{*}\right\}= & \arg \min _{w, l}\left\{\overline{x_{t}^{2}}+w^{2}\left(\left(\tilde{h}_{t}+\epsilon\right)^{2} \overline{x_{t}^{2}}+\sigma_{n}^{2}\right)+l^{2}\right. \\
& \left.-2 l \overline{x_{t}}+2 w l\left(\tilde{h}_{t}+\epsilon\right) \overline{x_{t}}-2 w\left(\tilde{h}_{t}+\epsilon\right) \overline{x_{t}^{2}}\right\} .
\end{aligned}
$$

We observe that the cost function in (11) is a convex function of $w$ and $l$ yielding

$w^{*}=\frac{\left(\tilde{h}_{t}+\epsilon\right) \sigma_{x}^{2}}{\left(\tilde{h}_{t}+\epsilon\right)^{2} \sigma_{x}^{2}+\sigma_{n}^{2}}, \quad l^{*}=\frac{\overline{x_{t}} \sigma_{n}^{2}}{\left(\tilde{h}_{t}+\epsilon\right)^{2} \sigma_{x}^{2}+\sigma_{n}^{2}}$.

However we have

$\frac{\overline{x_{t}^{2}}-l^{*} \overline{x_{t}}}{w^{*} \overline{x_{t}^{2}}}=\tilde{h}_{t}+\epsilon+\frac{\sigma_{n}^{2}}{\left(\tilde{h}_{t}+\epsilon\right) \sigma_{x}^{2}}>\tilde{h}_{t}$

so that $\left[w^{*}, l^{*}\right]^{T} \notin \mathcal{U}$.

We next substitute $\delta h_{t}=-\epsilon$ in the cost function of (4) to get

$$
\begin{aligned}
\left\{w^{*}, l^{*}\right\}= & \arg \min _{w, l}\left\{\overline{x_{t}^{2}}+w^{2}\left(\left(\tilde{h}_{t}-\epsilon\right)^{2} \overline{x_{t}^{2}}+\sigma_{n}^{2}\right)+l^{2}-2 l \overline{x_{t}}\right. \\
& \left.+2 w l\left(\tilde{h}_{t}-\epsilon\right) \overline{x_{t}}-2 w\left(\tilde{h}_{t}-\epsilon\right) \overline{x_{t}^{2}}\right\} .
\end{aligned}
$$

The cost function in (13) is also a convex function of $w$ and $l$ so that we get

$$
w^{*}=\frac{\left(\tilde{h}_{t}-\epsilon\right) \sigma_{x}^{2}}{\left(\tilde{h}_{t}-\epsilon\right)^{2} \sigma_{x}^{2}+\sigma_{n}^{2}}, \quad l^{*}=\frac{\overline{x_{t}} \sigma_{n}^{2}}{\left(\tilde{h}_{t}-\epsilon\right)^{2} \sigma_{x}^{2}+\sigma_{n}^{2}} .
$$

If the condition $\tilde{h}_{t} \epsilon \sigma_{x}^{2}<\epsilon^{2} \sigma_{x}^{2}+\sigma_{n}^{2}$ holds, then we have

$$
\tilde{h}_{t}<\tilde{h}_{t}-\epsilon+\frac{\sigma_{n}^{2}}{\left(\tilde{h}_{t}-\epsilon\right) \overline{x_{t}^{2}}}<\frac{\overline{x_{t}^{2}}-l \overline{x_{t}}}{w \overline{x_{t}^{2}}}
$$

so that $\left[w^{*}, l^{*}\right]^{T} \in \mathcal{V}$. Thus, the corresponding equalizer coefficient and the bias term are given by $w_{1, t}=\frac{\left(\tilde{h}_{t}-\epsilon\right) \sigma_{x}^{2}}{\left(\tilde{h}_{t}-\epsilon\right)^{2} \sigma_{x}^{2}+\sigma_{n}^{2}}$ and $l_{1, t}=\frac{\overline{x_{t}} \sigma_{n}^{2}}{\left(\tilde{h}_{t}-\epsilon\right)^{2} \sigma_{x}^{2}+\sigma_{n}^{2}}$, respectively. However, if the condition $\tilde{h}_{t} \epsilon \sigma_{x}^{2}<$ $\epsilon^{2} \sigma_{x}^{2}+\sigma_{n}^{2}$ does not hold, then it follows that $\tilde{h}_{t}+\frac{l \overline{x_{t}}-\overline{x_{t}^{2}}}{w \bar{x}_{t}^{2}}=0$, which implies that

$\tilde{h}_{t}=-\frac{l \overline{x_{t}}-\overline{x_{t}^{2}}}{w \overline{x_{t}^{2}}}$.

From (8), we observe that

$$
\begin{aligned}
E & {\left[\left(x_{t}-w\left(\left(\tilde{h}_{t}+\delta h_{t}\right) x_{t}+n_{t}\right)-l\right)^{2}\right] } \\
& =w^{2} \Delta h_{t}^{2} \overline{x_{t}^{2}}+2 w \Delta h_{t}\left(l \overline{x_{t}}-\overline{x_{t}^{2}}\right)+\mathrm{C}_{1} \\
& =w^{2} \overline{x_{t}^{2}}\left[\Delta h_{t}^{2}+2 \Delta h_{t}\left(\frac{l \overline{x_{t}}-\overline{x_{t}^{2}}}{w \overline{x_{t}^{2}}}\right)\right]+\mathrm{C}_{1} \\
& =w^{2} \overline{x_{t}^{2}}\left[\Delta h_{t}^{2}-2 \Delta h_{t} \tilde{h}_{t}\right]+\mathrm{C}_{1}
\end{aligned}
$$

where (15) follows from (14). If we add and subtract $w^{2} \bar{x}_{t}^{2} \tilde{h}_{t}^{2}$ to (15), then we get

$$
\begin{aligned}
& E\left[\left(x_{t}-w\left(\left(\tilde{h}_{t}+\delta h_{t}\right) x_{t}+n_{t}\right)-l\right)^{2}\right] \\
& \quad=w^{2} \overline{x_{t}^{2}}\left[\Delta h_{t}^{2}-2 \Delta h_{t} \tilde{h}_{t}+\tilde{h}_{t}^{2}\right]-w^{2} \bar{x}_{t}^{2} \tilde{h}_{t}^{2}+C_{1} \\
& \quad=w^{2} \bar{x}_{t}^{2} \delta h_{t}^{2}-w^{2} \bar{x}_{t}^{2} \tilde{h}_{t}^{2}+C_{1} .
\end{aligned}
$$

Here, if we maximize (16) with respect to $\delta h_{t}$, then it yields that the maximizer $\delta h_{t}^{*}$ is equal to $\epsilon$ or $-\epsilon$ so that

$$
\begin{aligned}
& \arg \max _{\left|\delta h_{t}\right| \leqslant \epsilon} E\left[\left(x_{t}-w\left(\left(\tilde{h}_{t}+\delta h_{t}\right) x_{t}+n_{t}\right)-l\right)^{2}\right] \\
& \quad=w^{2} \bar{x}_{t}^{2} \epsilon^{2}-w^{2} \overline{x_{t}^{2}} \tilde{h}_{t}^{2}+C_{1} \\
& \quad=w^{2} \overline{x_{t}^{2}}\left(\epsilon^{2}-\tilde{h}_{t}^{2}\right)+\overline{x_{t}^{2}}+w^{2} \sigma_{n}^{2}+l^{2}-2 l \overline{x_{t}} .
\end{aligned}
$$

If we take the derivative of (17) with respect to $l$ and equate it to zero, then it yields

$l_{1, t}=\overline{x_{t}}$

We next substitute $l_{1, t}$ into (14) to get

$w_{1, t}=\frac{\sigma_{x}^{2}}{\bar{x}_{t}^{2} \tilde{h}_{t}}$.

Hence, we have

$w_{1, t}= \begin{cases}\frac{\left(\tilde{h}_{t}-\epsilon\right) \sigma_{x}^{2}}{\left(\tilde{h}_{t}-\epsilon\right)^{2} \sigma_{x}^{2}+\sigma_{n}^{2}}: & \tilde{h}_{t} \epsilon \sigma_{x}^{2}<\epsilon^{2} \sigma_{x}^{2}+\sigma_{n}^{2}, \\ \frac{\sigma_{x}^{2}}{\bar{x}_{t}^{2} \tilde{h}_{t}}: & \tilde{h}_{t} \epsilon \sigma_{x}^{2} \geqslant \epsilon^{2} \sigma_{x}^{2}+\sigma_{n}^{2},\end{cases}$

$l_{1, t}= \begin{cases}\frac{\overline{x_{t}} \sigma_{n}^{2}}{\left(\tilde{h}_{t}-\epsilon\right)^{2} \sigma_{x}^{2}+\sigma_{n}^{2}}: & \tilde{h}_{t} \epsilon \sigma_{x}^{2}<\epsilon^{2} \sigma_{x}^{2}+\sigma_{n}^{2}, \\ \overline{x_{t}}: & \tilde{h}_{t} \in \sigma_{x}^{2} \geqslant \epsilon^{2} \sigma_{x}^{2}+\sigma_{n}^{2} .\end{cases}$

The proof follows.

In the following corollary, we provide a special case of Theorem 1 , where the desired signal $x_{t}$ is zero mean.

Corollary 1. When the transmitted signal $x_{t}$ is zero mean, the solution to the optimization problem (4) is given by 
$w_{1, t}= \begin{cases}\frac{\left(\tilde{h}_{t}-\epsilon\right)}{\left(\tilde{h}_{t}-\epsilon\right)^{2}+\frac{1}{S}}: & \epsilon\left(\tilde{h}_{t}-\epsilon\right)<\frac{1}{S}, \\ \frac{1}{\tilde{h}_{t}}: & \epsilon\left(\tilde{h}_{t}-\epsilon\right) \geqslant \frac{1}{S},\end{cases}$

where $S \triangleq \sigma_{x}^{2} / \sigma_{n}^{2}$ is the signal-to-noise ratio (SNR).

Proof. The proof directly follows from Theorem 1, therefore, is omitted.

\subsection{Affine equalization using a minimin framework}

In this section, we study the minimin equalization framework, where the inner maximization of the minimax framework is replaced with a minimization over the uncertainty set $[6,20,10]$. We seek to solve the optimization problem (5).

The following lemma is introduced to demonstrate that min operators in (5) can be interchanged, which will be used in Theorem 2.

Lemma 1. For an arbitrary function $f(x, y, z)$ and nonempty sets $\mathcal{X}, \mathcal{Y}$ and $\mathcal{Z}$, we have

$\min _{x \in \mathcal{X}, y \in \mathcal{Y}} \min _{z \in \mathcal{Z}} f(x, y, z)=\min _{z \in \mathcal{Z}} \min _{x \in \mathcal{X}, y \in \mathcal{X}} f(x, y, z)$,

assuming that all minimums are achieved on the corresponding sets.

Proof. The proof is given in the footnote. ${ }^{1}$

In the following theorem we present a closed-form solution to the optimization problem (5).

Theorem 2. Let $x_{t}, y_{t}$ and $n_{t}$ represent the transmitted, received and noise signals such that $y_{t}=h_{t} x_{t}+n_{t}$, where $h_{t}$ is the unknown channel coefficient and $n_{t}$ is i.i.d. zero mean with variance $\sigma_{n}^{2}$. At each time $t$, given an estimate $\tilde{h}_{t}$ of $h_{t}$ satisfying $\left|h_{t}-\tilde{h}_{t}\right| \leqslant \epsilon$, the solution to the optimization problem (5) is given by

$w_{2, t}=\frac{\left(\tilde{h}_{t}+\epsilon \operatorname{sign}\left(\tilde{h}_{t}\right)\right) \sigma_{x}^{2}}{\left(\tilde{h}_{t}+\epsilon \operatorname{sign}\left(\tilde{h}_{t}\right)\right)^{2} \sigma_{x}^{2}+\sigma_{n}^{2}}$

and

$l_{2, t}=\frac{\overline{x_{t}} \sigma_{n}^{2}}{\left(\tilde{h}_{t}+\epsilon \operatorname{sign}\left(\tilde{h}_{t}\right)\right) \sigma_{x}^{2}+\sigma_{n}^{2}}$,

where $\overline{x_{t}} \triangleq E\left[x_{t}\right]$ and $\sigma_{x}^{2} \triangleq E\left[\left(x_{t}-\overline{x_{t}}\right)^{2}\right]$ are the mean and variance of the transmitted signal $x_{t}$, respectively.

Proof. Here, we find the equalizer coefficient $w_{2, t}$ and the bias term $l_{2, t}$ that solve the optimization problem in (5). We first note that, by Lemma 1 , we can interchange min operators in (5) so that the optimization problem in (5) is equivalent to

$$
\begin{aligned}
& \min _{w, l} \min _{\left|\delta h_{t}\right| \leqslant \epsilon} E\left[\left(x_{t}-w\left(\left(\tilde{h}_{t}+\delta h_{t}\right) x_{t}+n_{t}\right)-l\right)^{2}\right] \\
& \quad=\min _{\left|\delta h_{t}\right| \leqslant \epsilon} \min _{w, l} E\left[\left(x_{t}-w\left(\left(\tilde{h}_{t}+\delta h_{t}\right) x_{t}+n_{t}\right)-l\right)^{2}\right] .
\end{aligned}
$$

\footnotetext{
1 To prove that $\min _{x \in \mathcal{X}}, y \in \mathcal{Y} \min _{z \in \mathcal{Z}} f(x, y, z)=\min _{z \in \mathcal{Z}} \min _{x \in \mathcal{X}, y \in \mathcal{X}} f(x, y, z)$, we first show that $\min _{x \in \mathcal{X}, y \in \mathcal{Y}} \min _{z \in \mathcal{Z}} f(x, y, z) \leqslant \min _{z \in \mathcal{Z}} \min _{x \in \mathcal{X}, y \in \mathcal{X}} f(x, y, z)$. We next show that $\min _{x \in \mathcal{X}, y \in \mathcal{Y}} \min _{z \in \mathcal{Z}} f(x, y, z) \geqslant \min _{z \in \mathcal{Z}} \min _{x \in \mathcal{X}, y \in \mathcal{X}} f(x, y, z)$. First, we observe that $\min _{z \in \mathcal{Z}} f(x, y, z) \leqslant f(x, y, z)$. Since this is true $\forall x \in \mathcal{X}$, $\forall y \in \mathcal{Y}$ and $\forall z \in \mathcal{X}$, it follows that $\min _{z \in \mathcal{Z}} f(x, y, z) \leqslant \min _{x \in \mathcal{X}}, y \in \mathcal{Y} f(x, y, z)$ $\forall x \in \mathcal{X}, \forall y \in \mathcal{Y}$ and $\forall z \in \mathcal{X}$. Therefore, we get that $\min _{x \in \mathcal{X}, y \in \mathcal{Y}} \min _{z \in \mathcal{Z}} f(x, y, z) \leqslant$ $\min _{x \in \mathcal{X}, y \in \mathcal{Y}} f(x, y, z) \forall z \in \mathcal{Z}$. Then, it follows that $\min _{x \in \mathcal{X}, y \in \mathcal{Y}} \min _{z \in \mathcal{Z}} f(x, y, z) \leqslant$ $\min _{z \in \mathcal{Z}} \min _{x \in \mathcal{X}, y \in \mathcal{X}} f(x, y, z)$. Using similar steps, it easily follows that the converse is also true. Hence, the proof follows.
}

Hence, we first solve the inner minimization problem in (18) and find the minimizers $w^{*}$ and $l^{*}$. We then substitute $w^{*}$ and $l^{*}$ in (18) and solve the outer minimization problem to find the minimizer $\delta h_{t}^{*}$, which yields the desired equalizer coefficient $w_{2, t}$ and $l_{2, t}$.

We observe that the objective function in (18) can be written as

$$
\begin{aligned}
& E\left[\left(x_{t}-w\left(\left(\tilde{h}_{t}+\delta h_{t}\right) x_{t}+n_{t}\right)-l\right)^{2}\right] \\
& \quad=\overline{x_{t}^{2}}+w^{2}\left(\Delta h_{t}^{2} \overline{x_{t}^{2}}+\sigma_{n}^{2}\right)+l^{2}-2 l \overline{x_{t}}+2 w l \Delta h_{t} \overline{x_{t}}-2 w \Delta h_{t} \overline{x_{t}^{2}},
\end{aligned}
$$

where $\overline{x_{t}^{2}} \triangleq E\left[x_{t}^{2}\right]$ and $\Delta h_{t} \triangleq \tilde{h}_{t}+\delta h_{t}$

We first solve the inner minimization problem in the right-hand side of (18) with respect to $w$ and $l$ as follows. We define $F(w, l)=$ $E\left[\left(x_{t}-w\left(\Delta h_{t} x_{t}+n_{t}\right)-l\right)^{2}\right]$. Note that $F(w, l)$ is a quadratic function of the variables $w$ and $l$ with positive leading term coefficients, i.e., the coefficients of $w^{2}$ and $l^{2}$ are positive. Hence, it is a convex function of the variables $w$ and $l$, which implies that it has a global minimum point $\left(w^{*}, l^{*}\right)$. If we set the first derivatives of $F(w, l)$ with respect to $w$ and $l$, then it yields the minimizers $w^{*}$ and $l^{*}$, respectively, i.e., $w^{*}$ and $l^{*}$ satisfy $\left.\frac{\partial F}{\partial w}\right|_{w=w^{*}}=0$ and $\left.\frac{\partial F}{\partial l}\right|_{l=l^{*}}=0$. The corresponding partial derivative of the cost function $F(w, l)$ with respect to $l$ is given by

$\left.\frac{\partial F}{\partial l}\right|_{l=l^{*}}=2 l^{*}-2 \overline{x_{t}}+2 w^{*} \Delta h_{t} \overline{x_{t}}=0$

so that $l^{*}=\overline{x_{t}}-w^{*} \Delta h_{t} \overline{x_{t}}$. The corresponding partial derivative of $F(w, l)$ with respect to $w$ is given by

$$
\left.\frac{\partial F}{\partial w}\right|_{w=w^{*}}=2 w^{*}\left(\Delta h_{t}^{2} \overline{x_{t}^{2}}+\sigma_{n}^{2}\right)+2 l^{*} \Delta h_{t} \overline{x_{t}}-2 \Delta h_{t} \overline{x_{t}^{2}}=0,
$$

which implies that $w^{*}=\frac{\Delta h_{t} \overline{x_{t}^{2}}-l^{*} \Delta h_{t} \overline{x_{t}}}{\Delta h_{t}^{2} \bar{x}_{t}^{2}+\sigma_{n}^{2}}$. Thus, we get that

$$
\begin{aligned}
& w^{*}=\frac{\Delta h_{t} \sigma_{x}^{2}}{\Delta h_{t}^{2} \sigma_{x}^{2}+\sigma_{n}^{2}}, \\
& l^{*}=\frac{\overline{x_{t}} \sigma_{n}^{2}}{\Delta h_{t}^{2} \sigma_{x}^{2}+\sigma_{n}^{2}}
\end{aligned}
$$

for a given $\delta h_{t}$.

We next solve the outer minimization problem. If we substitute $w^{*}$ and $l^{*}$ in $F(w, l)$, then we obtain

$$
\begin{aligned}
\delta h_{t}^{*} & =\arg \min _{\left|\delta h_{t}\right| \leqslant \epsilon} \min _{w, l} E\left[\left(x_{t}-w\left(\left(\tilde{h}_{t}+\delta h_{t}\right) x_{t}+n_{t}\right)-l\right)^{2}\right] \\
& =\arg \min _{\left|\delta h_{t}\right| \leqslant \epsilon} F\left(w^{*}, l^{*}\right) \\
& =\arg \min _{\left|\delta h_{t}\right| \leqslant \epsilon} \frac{\sigma_{n}^{2} \sigma_{x}^{2}}{\left(\tilde{h}_{t}+\delta h_{t}\right)^{2} \sigma_{x}^{2}+\sigma_{n}^{2}} \\
& =\arg \max _{\left|\delta h_{t}\right| \leqslant \epsilon}\left|\tilde{h}_{t}+\delta h_{t}\right|
\end{aligned}
$$

so that $\delta h_{t}^{*}=\epsilon \operatorname{sign}\left(\tilde{h}_{t}\right)$. Hence, the equalizer coefficient $w_{2, t}$ and the bias term $l_{2, t}$ are given by

$$
\begin{aligned}
& w_{2, t}=\frac{\left(\tilde{h}_{t}+\epsilon \operatorname{sign}\left(\tilde{h}_{t}\right)\right) \sigma_{x}^{2}}{\left(\tilde{h}_{t}+\epsilon \operatorname{sign}\left(\tilde{h}_{t}\right)\right)^{2} \sigma_{x}^{2}+\sigma_{n}^{2}}, \\
& l_{2, t}=\frac{\overline{x_{t}} \sigma_{n}^{2}}{\left(\tilde{h}_{t}+\epsilon \operatorname{sign}\left(\tilde{h}_{t}\right)\right) \sigma_{x}^{2}+\sigma_{n}^{2}} .
\end{aligned}
$$

Hence, the proof follows. 
In the following corollary, we provide a special case of Theorem 1 , where the desired signal $x_{t}$ is zero mean.

Corollary 2. When the transmitted signal $x_{t}$ is zero mean, the solution to the optimization problem (5) is given by

$w_{2, t}=\frac{\left(\tilde{h}_{t}+\epsilon \operatorname{sign}\left(\tilde{h}_{t}\right)\right)}{\left(\tilde{h}_{t}+\epsilon \operatorname{sign}\left(\tilde{h}_{t}\right)\right)^{2}+\frac{1}{S}}$

and

$l_{2, t}=0$,

where $S \triangleq \sigma_{x}^{2} / \sigma_{n}^{2}$ is the $S N R$.

Proof. The proof follows from Theorem 2 when $\overline{x_{t}}=0$.

\subsection{Affine equalization using a minimax regret framework}

In this section, we investigate the minimax regret equalization framework, where the performance of an affine equalizer is defined with respect to the MMSE affine equalizer that is tuned to the unknown channel $[4,7,11,16]$. We emphasize that the minimax equalization framework investigated in Section 3.2 may produce highly conservative results since the equalizer coefficient $w$ and the bias term $l$ are optimized to minimize the worst case MSE [16]. Moreover, the minimin equalization framework introduced in Section 3.3 is a highly optimistic method where the equalizer parameters are optimized to minimize the MSE that corresponds to the most favorable channel [6]. Thus, the minimin approach may also yield unsatisfactory results in certain applications, where the channel estimate is highly erroneous [6]. In this context, the minimax regret equalization framework can be used to improve the equalization performance while preserving the robustness [4,7]. In this approach, we find the equalizer coefficient $w_{3, t}$ and the bias term $l_{3, t}$ that solve the optimization problem (6).

We note that from Section 3.3, the solution to the minimization problem in the objective function is given by

$\min _{w, l} E\left[\left(x_{t}-w\left(\left(\tilde{h}_{t}+\delta h_{t}\right) x_{t}+n_{t}\right)-l\right)^{2}\right]=\frac{\sigma_{n}^{2} \sigma_{x}^{2}}{\left(\tilde{h}_{t}+\delta h_{t}\right)^{2} \sigma_{x}^{2}+\sigma_{n}^{2}}$,

where $\sigma_{x}^{2} \triangleq E\left[\left(x_{t}-\overline{x_{t}}\right)^{2}\right]$ is the variance of the transmitted signal $x_{t}$. Hence the optimization problem in (6) is equivalent to

$$
\begin{aligned}
& \arg \min _{w, l} \max _{\left|\delta h_{t}\right| \leqslant \epsilon}\left\{E\left[\left(x_{t}-w\left(\left(\tilde{h}_{t}+\delta h_{t}\right) x_{t}+n_{t}\right)-l\right)^{2}\right]\right. \\
& \left.\quad-\min _{w, l} E\left[\left(x_{t}-w\left(\left(\tilde{h}_{t}+\delta h_{t}\right) x_{t}+n_{t}\right)-l\right)^{2}\right]\right\} \\
& =\arg \min _{w, l} \max _{\left|\delta h_{t}\right| \leqslant \epsilon}\left\{E\left[\left(x_{t}-w\left(\left(\tilde{h}_{t}+\delta h_{t}\right) x_{t}+n_{t}\right)-l\right)^{2}\right]\right. \\
& \left.\quad-\frac{\sigma_{n}^{2} \sigma_{x}^{2}}{\left(\tilde{h}_{t}+\delta h_{t}\right)^{2}+\sigma_{n}^{2}}\right\} .
\end{aligned}
$$

We first expand the term

$$
\frac{\sigma_{n}^{2} \sigma_{x}^{2}}{\left(\tilde{h}_{t}+\delta h_{t}\right)^{2} \sigma_{x}^{2}+\sigma_{n}^{2}}
$$

in (20) around $\delta h_{t}=0$ yielding

$$
\frac{\sigma_{n}^{2} \sigma_{x}^{2}}{\left(\tilde{h}_{t}+\delta h_{t}\right)^{2}+\sigma_{n}^{2}} \approx \frac{\sigma_{n}^{2} \sigma_{x}^{2}}{\tilde{h}_{t}^{2}+\sigma_{n}^{2}}-\delta h_{t} \frac{2 \tilde{h}_{t} \sigma_{n}^{2} \sigma_{x}^{4}}{\left(\tilde{h}_{t}^{2} \sigma_{x}^{2}+\sigma_{n}^{2}\right)^{2}} .
$$

Hence, instead of (6), we solve the following optimization problem:

$$
\begin{aligned}
\left\{w_{3, t}, l_{3, t}\right\}= & \arg \min _{w, l} \max _{\left|\delta h_{t}\right| \leqslant \epsilon}\left\{E\left[\left(x_{t}-w\left(\left(\tilde{h}_{t}+\delta h_{t}\right) x_{t}+n_{t}\right)-l\right)^{2}\right]\right. \\
& \left.-\frac{\sigma_{n}^{2} \sigma_{x}^{2}}{\tilde{h}_{t}^{2}+\sigma_{n}^{2}}+\delta h_{t} \frac{2 \tilde{h}_{t} \sigma_{n}^{2} \sigma_{x}^{4}}{\left(\tilde{h}_{t}^{2} \sigma_{x}^{2}+\sigma_{n}^{2}\right)^{2}}\right\},
\end{aligned}
$$

which provides satisfactory results even under large derivations $\delta h_{t}$ as shown in the Simulations section.

In the following theorem we present a closed-form solution to the optimization problem (21).

Theorem 3. Let $x_{t}, y_{t}$ and $n_{t}$ represent the transmitted, received and noise signals such that $y_{t}=h_{t} x_{t}+n_{t}$, where $h_{t}$ is the unknown channel coefficient and $n_{t}$ is i.i.d. zero mean with variance $\sigma_{n}^{2}$. At each time $t$, given an estimate $\tilde{h}_{t}$ of $h_{t}$ satisfying $\left|h_{t}-\tilde{h}_{t}\right| \leqslant \epsilon$, the solution to the optimization problem (21) is given by

$\left[w_{3, t}, l_{3, t}\right]= \begin{cases}{\left[w_{1}^{*}, l_{1}^{*}\right]:} & f \geqslant 0, g \geqslant 0, \\ {\left[w_{2}^{*}, l_{2}^{*}\right]:} & f \leqslant 0, g \leqslant 0, \\ {\left[w_{3}^{*}, l_{3}^{*}\right]:} & f \geqslant 0, g \leqslant 0, \\ {\left[w_{4}^{*}, l_{4}^{*}\right]:} & f<0, g>0,\end{cases}$

where

$$
\begin{aligned}
& {\left[w_{1}^{*}, l_{1}^{*}\right]=} {\left[\frac{\left(\tilde{h}_{t}+\epsilon\right) \sigma_{x}^{2}}{\left(\tilde{h}_{t}+\epsilon\right)^{2} \sigma_{x}^{2}+\sigma_{n}^{2}}, \frac{\overline{x_{t}} \sigma_{n}^{2}}{\left(\tilde{h}_{t}+\epsilon\right)^{2} \sigma_{x}^{2}+\sigma_{n}^{2}}\right], } \\
& {\left[w_{2}^{*}, l_{2}^{*}\right]=} {\left[\frac{\left(\tilde{h}_{t}-\epsilon\right) \sigma_{x}^{2}}{\left(\tilde{h}_{t}-\epsilon\right)^{2} \sigma_{x}^{2}+\sigma_{n}^{2}}, \frac{\overline{x_{t}} \sigma_{n}^{2}}{\left(\tilde{h}_{t}-\epsilon\right)^{2} \sigma_{x}^{2}+\sigma_{n}^{2}}\right], } \\
& {\left[w_{3}^{*}, l_{3}^{*}\right]=} \arg \min _{[w, l] \in\left\{\left[w_{1}^{*}, l_{1}^{*}\right],\left[w_{2}^{*}, l_{2}^{*}\right]\right\}} \\
& \times\left\{\max _{\left|\delta h_{t}\right| \leqslant \epsilon}\left\{E\left[\left(x_{t}-w\left(\tilde{h}_{t}+\delta h_{t}\right) x_{t}+n_{t}\right)-l\right)^{2}\right]\right. \\
&\left.\left.-\frac{\sigma_{n}^{2} \sigma_{x}^{2}}{\tilde{h}_{t}^{2}+\sigma_{n}^{2}}+\delta h_{t} \frac{2 \tilde{h}_{t} \sigma_{n}^{2} \sigma_{x}^{4}}{\left(\tilde{h}_{t}^{2} \sigma_{x}^{2}+\sigma_{n}^{2}\right)^{2}}\right\}\right\}, \\
&\left.w_{4}^{*}, l_{4}^{*}\right]= \arg \min _{[w, l]}\left\{E\left[\left(x_{t}-w\left(\tilde{h}_{t} x_{t}+n_{t}\right)-l\right)^{2}\right]-\frac{\sigma_{n}^{2} \sigma_{x}^{2}}{\tilde{h}_{t}^{2}+\sigma_{n}^{2}}\right\}, \\
& f \triangleq-\epsilon- \frac{\bar{x}_{t}^{2} \sigma_{n}^{2}}{\left(\tilde{h}_{t}+\epsilon\right)^{2} \sigma_{x}^{2}+\sigma_{n}^{2}}-\frac{\sigma_{n}^{2}}{\left(\tilde{h}_{t}+\epsilon\right) \sigma_{x}^{2}} \\
& g \triangleq \epsilon-\frac{\tilde{h}_{t} \sigma_{n}^{2}}{\left(\tilde{h}_{t}+\epsilon\right)^{2}}\left(\frac{\left(\tilde{h}_{t}+\epsilon\right)^{2} \sigma_{x}^{2}+\sigma_{n}^{2}}{\tilde{h}_{t}^{2} \sigma_{x}^{2}+\sigma_{n}^{2}},\right. \frac{\bar{x}_{t}^{2} \sigma_{n}^{2}}{\left(\tilde{h}_{t}-\epsilon\right)^{2} \sigma_{x}^{2}+\sigma_{n}^{2}}-\frac{\sigma_{n}^{2}}{\left(\tilde{h}_{t}-\epsilon\right) \sigma_{x}^{2}} \\
&+\frac{\tilde{h}_{t} \sigma_{n}^{2}}{\left(\tilde{h}_{t}-\epsilon\right)^{2}}\left(\frac{\left(\tilde{h}_{t}-\epsilon\right)^{2} \sigma_{x}^{2}+\sigma_{n}^{2}}{\tilde{h}_{t}^{2} \sigma_{x}^{2}+\sigma_{n}^{2}}\right)^{2} .
\end{aligned}
$$

Here, $\overline{x_{t}} \triangleq E\left[x_{t}\right]$ and $\sigma_{x}^{2} \triangleq E\left[\left(x_{t}-\overline{x_{t}}\right)^{2}\right]$ are the mean and variance of the transmitted signal $x_{t}$, respectively.

Proof. We first observe that the objective function in (20) can be written as

$$
\begin{aligned}
& E\left[\left(x_{t}-w\left(\left(\tilde{h}_{t}+\delta h_{t}\right) x_{t}+n_{t}\right)-l\right)^{2}\right]-\frac{\sigma_{n}^{2} \sigma_{x}^{2}}{\tilde{h}_{t}^{2}+\sigma_{n}^{2}} \\
& \quad+\delta h_{t} \frac{2 \tilde{h}_{t} \sigma_{n}^{2} \sigma_{x}^{4}}{\left(\tilde{h}_{t}^{2} \sigma_{x}^{2}+\sigma_{n}^{2}\right)^{2}} \\
& =w^{2} \Delta h_{t}^{2} \overline{x_{t}^{2}}+\Delta h_{t}\left(2 w l \overline{x_{t}}-2 w \overline{x_{t}^{2}}+\frac{2 \tilde{h}_{t} \sigma_{n}^{2} \sigma_{x}^{4}}{\left(\tilde{h}_{t}^{2} \sigma_{x}^{2}+\sigma_{n}^{2}\right)^{2}}\right) \\
& \quad+\mathrm{D}_{1},
\end{aligned}
$$


where $\overline{x_{t}^{2}} \triangleq E\left[x_{t}^{2}\right], \Delta h_{t} \triangleq \tilde{h}_{t}+\delta h_{t}, \mathrm{D}_{1} \triangleq \overline{x_{t}^{2}}+w^{2} \sigma_{n}^{2}+l^{2}-2 l \overline{x_{t}}-$ $\frac{\sigma_{n}^{2} \sigma_{x}^{2}}{\tilde{h}_{t}^{2}+\sigma_{n}^{2}}-\tilde{h}_{t} \frac{2 \tilde{h}_{t} \sigma_{n}^{2} \sigma_{x}^{4}}{\left(\tilde{h}_{t}^{2} \sigma_{x}^{2}+\sigma_{n}^{2}\right)^{2}}$ is independent of $\delta h_{t}$. If we define $a=$ $w^{2} \overline{x_{t}^{2}} \geqslant 0, b \triangleq 2 w \overline{x_{t}}-2 w \overline{x_{t}^{2}}+\frac{2 \tilde{h}_{t} \sigma_{n}^{2} \sigma_{x}^{4}}{\left(\tilde{h}_{t}^{2} \sigma_{x}^{2}+\sigma_{n}^{2}\right)^{2}}$ and $\mathrm{D}_{2}=\mathrm{D}_{1}-\frac{b^{2}}{4 a}$, then (22) can be written as

$$
\begin{array}{r}
E\left[\left(x_{t}-w\left(\left(\tilde{h}_{t}+\delta h_{t}\right) x_{t}+n_{t}\right)-l\right)^{2}\right]-\frac{\sigma_{n}^{2} \sigma_{x}^{2}}{\tilde{h}_{t}^{2}+\sigma_{n}^{2}} \\
\quad+\delta h_{t} \frac{2 \tilde{h}_{t} \sigma_{n}^{2} \sigma_{x}^{4}}{\left(\tilde{h}_{t}^{2} \sigma_{x}^{2}+\sigma_{n}^{2}\right)^{2}}=a\left(u+\frac{b}{2 a}\right)^{2}+\mathrm{D}_{2},
\end{array}
$$

where $\mathrm{D}_{2}$ is independent of $\delta h_{t}$. Hence, the inner maximization problem in (21) is given by

$$
\begin{aligned}
\delta h_{t}^{*}= & \arg \max _{\left|\delta h_{t}\right| \leqslant \epsilon}\left\{E\left[\left(x_{t}-w\left(\left(\tilde{h}_{t}+\delta h_{t}\right) x_{t}+n_{t}\right)-l\right)^{2}\right]\right. \\
& \left.-\frac{\sigma_{n}^{2} \sigma_{x}^{2}}{\tilde{h}_{t}^{2}+\sigma_{n}^{2}}+\delta h_{t} \frac{2 \tilde{h}_{t} \sigma_{n}^{2} \sigma_{x}^{4}}{\left(\tilde{h}_{t}^{2} \sigma_{x}^{2}+\sigma_{n}^{2}\right)^{2}}\right\} \\
= & \arg \max _{\left|\delta h_{t}\right| \leqslant \epsilon}\left|\delta h_{t}+\tilde{h}_{t}+\frac{l \overline{x_{t}}}{w \overline{x_{t}^{2}}}-\frac{1}{w}+\frac{\tilde{h}_{t} \sigma_{n}^{2} \sigma_{x}^{4}}{w^{2} \overline{x_{t}^{2}}\left(\tilde{h}_{t}^{2} \sigma_{x}^{2}+\sigma_{n}^{2}\right)^{2}}\right| .
\end{aligned}
$$

By applying the triangular inequality to the cost function in (23), we get the following upper bound:

$$
\begin{aligned}
& \left|\delta h_{t}+\tilde{h}_{t}+\frac{l \overline{x_{t}}}{w \overline{x_{t}^{2}}}-\frac{1}{w}+\frac{\tilde{h}_{t} \sigma_{n}^{2} \sigma_{x}^{4}}{w^{2} \overline{x_{t}^{2}}\left(\tilde{h}_{t}^{2} \sigma_{x}^{2}+\sigma_{n}^{2}\right)^{2}}\right| \\
& \leqslant\left|\delta h_{t}\right|+\left|\tilde{h}_{t}+\frac{l \overline{x_{t}}}{w \overline{x_{t}^{2}}}-\frac{1}{w}+\frac{\tilde{h}_{t} \sigma_{n}^{2} \sigma_{x}^{4}}{w^{2} \overline{x_{t}^{2}}\left(\tilde{h}_{t}^{2} \sigma_{x}^{2}+\sigma_{n}^{2}\right)^{2}}\right| \\
& \leqslant \epsilon+\left|\tilde{h}_{t}+\frac{l \overline{x_{t}}}{w \overline{x_{t}^{2}}}-\frac{1}{w}+\frac{\tilde{h}_{t} \sigma_{n}^{2} \sigma_{x}^{4}}{w^{2} \overline{x_{t}^{2}}\left(\tilde{h}_{t}^{2} \sigma_{x}^{2}+\sigma_{n}^{2}\right)^{2}}\right|,
\end{aligned}
$$

where the upper bound is achieved at $\delta h_{t}^{*}=\epsilon \operatorname{sgn}\left(\tilde{h}_{t}+\frac{l \overline{x_{t}}}{w x_{t}^{2}}-\frac{1}{w}+\right.$ $\left.\frac{\tilde{h}_{t} \sigma_{n}^{2} \sigma_{x}^{4}}{w^{2} x_{t}^{2}\left(\tilde{h}_{t}^{2} \sigma_{x}^{2}+\sigma_{n}^{2}\right)^{2}}\right)$. Hence it follows that

$$
\begin{aligned}
& \delta h_{t}^{*}=\arg \max _{\left|\delta h_{t}\right| \leqslant \epsilon}\left\{E\left[\left(x_{t}-w\left(\left(\tilde{h}_{t}+\delta h_{t}\right) x_{t}+n_{t}\right)-l\right)^{2}\right]\right. \\
& \left.-\frac{\sigma_{n}^{2} \sigma_{x}^{2}}{\tilde{h}_{t}^{2}+\sigma_{n}^{2}}+\delta h_{t} \frac{2 \tilde{h}_{t} \sigma_{n}^{2} \sigma_{x}^{4}}{\left(\tilde{h}_{t}^{2} \sigma_{x}^{2}+\sigma_{n}^{2}\right)^{2}}\right\}
\end{aligned}
$$

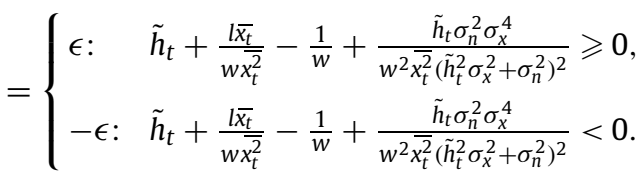

We next solve the outer minimization problem as follows. If we write $\boldsymbol{u}=[w, l]^{T} \in \mathbb{R}^{2}$ and define $\mathcal{M}=\left\{\boldsymbol{u}=[w, l]^{T} \in \mathbb{R}^{2}\right.$ |

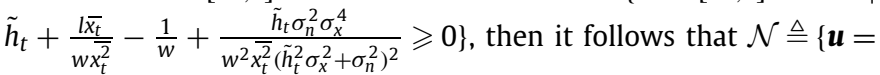
$\left.[w, l]^{T} \in \mathbb{R}^{2} \mid \tilde{h}_{t}+\frac{l \overline{x_{t}}}{w \overline{x_{t}^{2}}}-\frac{1}{w}+\frac{\tilde{h}_{t} \sigma_{n}^{2} \sigma_{x}^{4}}{w^{2} \bar{x}_{t}^{2}\left(\tilde{h}_{t}^{2} \sigma_{x}^{2}+\sigma_{n}^{2}\right)^{2}}<0\right\}=\mathbb{R}^{2} \backslash \mathcal{M}$, i.e., $\mathcal{M} \cup \mathcal{N}=\mathbb{R}^{2}$ and $\mathcal{M} \cap \mathcal{N}=\varnothing$. Hence, the cost function in the outer minimization problem in (21) is given by

$$
\begin{gathered}
\max _{\left|\delta h_{t}\right| \leqslant \epsilon}\left\{E\left[\left(x_{t}-w\left(\left(\tilde{h}_{t}+\delta h_{t}\right) x_{t}+n_{t}\right)-l\right)^{2}\right]\right. \\
\left.-\frac{\sigma_{n}^{2} \sigma_{x}^{2}}{\tilde{h}_{t}^{2}+\sigma_{n}^{2}}+\delta h_{t} \frac{2 \tilde{h}_{t} \sigma_{n}^{2} \sigma_{x}^{4}}{\left(\tilde{h}_{t}^{2} \sigma_{x}^{2}+\sigma_{n}^{2}\right)^{2}}\right\}
\end{gathered}
$$

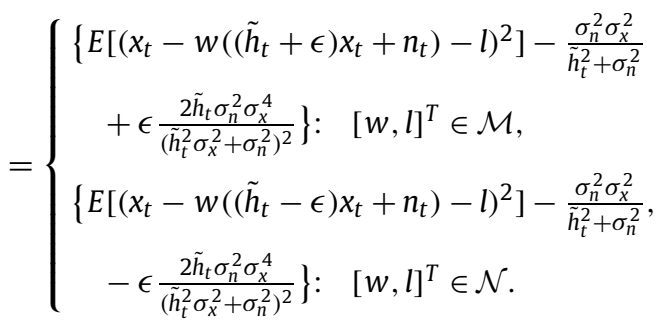

We first substitute $\delta h_{t}=\epsilon$ and find the corresponding $\{w, l\}$ pair that minimizes the objective function in (21) to check whether $[w, l] \in \mathcal{M}$. We next substitute $\delta h_{t}=-\epsilon$ and find the corresponding $\{w, l\}$ to check whether $[w, l] \in \mathcal{N}$. Based on these criteria, we obtain the corresponding equalizer coefficient and the bias term pair $\left\{w_{3, t}, l_{3, t}\right\}$.

We first substitute $\delta h_{t}=\epsilon$ in the cost function in (21) to get the following minimization problem:

$$
\begin{aligned}
\left\{w_{1}^{*}, l_{1}^{*}\right\}= & \arg \min _{w, l}\left\{\overline{x_{t}^{2}}+w^{2}\left(\tilde{h}_{t}+\epsilon\right)^{2} \overline{x_{t}^{2}}+w^{2} \sigma_{n}^{2}+l^{2}\right. \\
& -2 w\left(\tilde{h}_{t}+\epsilon\right) \overline{x_{t}^{2}}-2 \overline{x_{t}} l-2 \overline{x_{t}} w\left(\tilde{h}_{t}+\epsilon\right) l \\
& \left.-\frac{\sigma_{n}^{2} \sigma_{x}^{2}}{\tilde{h}_{t}^{2}+\sigma_{n}^{2}}+\epsilon \frac{2 \tilde{h}_{t} \sigma_{n}^{2} \sigma_{x}^{4}}{\left(\tilde{h}_{t}^{2} \sigma_{x}^{2}+\sigma_{n}^{2}\right)^{2}}\right\} .
\end{aligned}
$$

Since the cost function in (25) is a convex function of $w$ and $l$, we get that

$$
w_{1}^{*}=\frac{\left(\tilde{h}_{t}+\epsilon\right) \sigma_{x}^{2}}{\left(\tilde{h}_{t}+\epsilon\right)^{2} \sigma_{x}^{2}+\sigma_{n}^{2}}, \quad l_{1}^{*}=\frac{\overline{x_{t}} \sigma_{n}^{2}}{\left(\tilde{h}_{t}+\epsilon\right)^{2} \sigma_{x}^{2}+\sigma_{n}^{2}} .
$$

We observe that $\left[w_{1}^{*}, l_{1}^{*}\right] \in \mathcal{M}$ if and only if

$$
\begin{aligned}
f \triangleq & -\epsilon-\frac{\bar{x}_{t}^{2} \sigma_{n}^{2}}{\left(\tilde{h}_{t}+\epsilon\right)^{2} \sigma_{x}^{2}+\sigma_{n}^{2}}-\frac{\sigma_{n}^{2}}{\left(\tilde{h}_{t}+\epsilon\right) \sigma_{x}^{2}} \\
& +\frac{\tilde{h}_{t} \sigma_{n}^{2}}{\left(\tilde{h}_{t}+\epsilon\right)^{2}}\left(\frac{\left(\tilde{h}_{t}+\epsilon\right)^{2} \sigma_{x}^{2}+\sigma_{n}^{2}}{\tilde{h}_{t}^{2} \sigma_{x}^{2}+\sigma_{n}^{2}}\right)^{2} \geqslant 0 .
\end{aligned}
$$

We next substitute $\delta h_{t}=-\epsilon$ in the cost function in (21) to get the following minimization problem:

$$
\begin{aligned}
\left\{w_{2}^{*}, l_{2}^{*}\right\}= & \arg \min _{w, l}\left\{\overline{x_{t}^{2}}+w^{2}\left(\tilde{h}_{t}-\epsilon\right)^{2} \overline{x_{t}^{2}}+w^{2} \sigma_{n}^{2}+l^{2}\right. \\
& -2 w\left(\tilde{h}_{t}-\epsilon\right) \overline{x_{t}^{2}}-2 \overline{x_{t}} l-2 \overline{x_{t}} w\left(\tilde{h}_{t}-\epsilon\right) l \\
& \left.-\frac{\sigma_{n}^{2} \sigma_{x}^{2}}{\tilde{h}_{t}^{2}+\sigma_{n}^{2}}-\epsilon \frac{2 \tilde{h}_{t} \sigma_{n}^{2} \sigma_{x}^{4}}{\left(\tilde{h}_{t}^{2} \sigma_{x}^{2}+\sigma_{n}^{2}\right)^{2}}\right\} .
\end{aligned}
$$

Since the cost function in (26) is a convex function of $w$ and $l$, we get that

$$
w_{2}^{*}=\frac{\left(\tilde{h}_{t}-\epsilon\right) \sigma_{x}^{2}}{\left(\tilde{h}_{t}-\epsilon\right)^{2} \sigma_{x}^{2}+\sigma_{n}^{2}}, \quad l_{2}^{*}=\frac{\overline{x_{t}} \sigma_{n}^{2}}{\left(\tilde{h}_{t}-\epsilon\right)^{2} \sigma_{x}^{2}+\sigma_{n}^{2}} .
$$

Note that $\left[w_{2}^{*}, l_{2}^{*}\right] \in \mathcal{N}$ if and only if

$$
\begin{aligned}
g \triangleq & -\frac{\bar{x}_{t}^{2} \sigma_{n}^{2}}{\left(\tilde{h}_{t}-\epsilon\right)^{2} \sigma_{x}^{2}+\sigma_{n}^{2}}-\frac{\sigma_{n}^{2}}{\left(\tilde{h}_{t}-\epsilon\right) \sigma_{x}^{2}} \\
& +\frac{\tilde{h}_{t} \sigma_{n}^{2}}{\left(\tilde{h}_{t}-\epsilon\right)^{2}}\left(\frac{\left(\tilde{h}_{t}-\epsilon\right)^{2} \sigma_{x}^{2}+\sigma_{n}^{2}}{\tilde{h}_{t}^{2} \sigma_{x}^{2}+\sigma_{n}^{2}}\right)^{2} \leqslant 0 .
\end{aligned}
$$
$\sigma_{n}^{2}$ :

There are four cases depending on the values of $\tilde{h}_{t}, \epsilon, \overline{x_{t}}, \overline{x_{t}^{2}}$, 


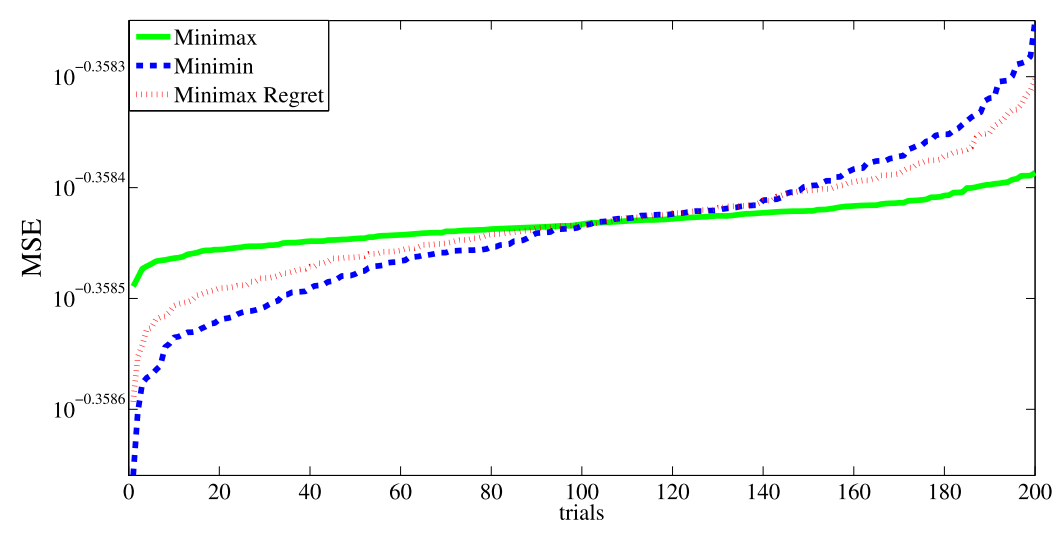

Fig. 2. Sorted MSEs for the minimax, minimin and minimax regret equalization methods over 200 trials when $\epsilon=0.3$.

- Case $1: f \geqslant 0$ and $g \geqslant 0$.

In this case, we have

$w_{3, t}=\frac{\left(\tilde{h}_{t}+\epsilon\right) \sigma_{x}^{2}}{\left(\tilde{h}_{t}+\epsilon\right)^{2} \sigma_{x}^{2}+\sigma_{n}^{2}}$

and

$l_{3, t}=\frac{\overline{x_{t}} \sigma_{n}^{2}}{\left(\tilde{h}_{t}+\epsilon\right)^{2} \sigma_{x}^{2}+\sigma_{n}^{2}}$

since $\left[w_{1}^{*}, l_{1}^{*}\right] \in \mathcal{M}$ and $\left[w_{2}^{*}, l_{2}^{*}\right] \notin \mathcal{N}$.

- Case 2: $f \leqslant 0$ and $g \leqslant 0$.

In this case, we have

$$
w_{3, t}=\frac{\left(\tilde{h}_{t}-\epsilon\right) \sigma_{x}^{2}}{\left(\tilde{h}_{t}-\epsilon\right)^{2} \sigma_{x}^{2}+\sigma_{n}^{2}}
$$

and

$$
l_{3, t}=\frac{\overline{x_{t}} \sigma_{n}^{2}}{\left(\tilde{h}_{t}-\epsilon\right)^{2} \sigma_{x}^{2}+\sigma_{n}^{2}}
$$

since $\left[w_{1}^{*}, l_{1}^{*}\right] \notin \mathcal{M}$ and $\left[w_{2}^{*}, l_{2}^{*}\right] \in \mathcal{N}$.

- Case 3: $f \geqslant 0$ and $g \leqslant 0$.

In this case, we have $\left[w_{1}^{*}, l_{1}^{*}\right] \in \mathcal{M}$ and $\left[w_{2}^{*}, l_{2}^{*}\right] \in \mathcal{N}$ so that

$$
\begin{aligned}
{\left[w_{3, t}, l_{3, t}\right]=} & \arg \min _{[w, l] \in\left\{\left[w_{1}^{*}, l_{1}^{*}\right],\left[w_{2}^{*}, l_{2}^{*}\right]\right\}}\left\{\operatorname { m a x } _ { | \delta h _ { t } | \leqslant \epsilon } \left\{E \left[\left(x_{t}\right.\right.\right.\right. \\
& \left.\left.-w\left(\left(\tilde{h}_{t}+\delta h_{t}\right) x_{t}+n_{t}\right)-l\right)^{2}\right] \\
& \left.\left.-\frac{\sigma_{n}^{2} \sigma_{x}^{2}}{\tilde{h}_{t}^{2}+\sigma_{n}^{2}}+\delta h_{t} \frac{2 \tilde{h}_{t} \sigma_{n}^{2} \sigma_{x}^{4}}{\left(\tilde{h}_{t}^{2} \sigma_{x}^{2}+\sigma_{n}^{2}\right)^{2}}\right\}\right\} .
\end{aligned}
$$

- Case 4: $f \leqslant 0$ and $g \geqslant 0$.

In the last case, we have the optimum points on the curve $\tilde{h}_{t}+\frac{l \overline{x_{t}}}{w \overline{x_{t}^{2}}}-\frac{1}{w}+\frac{\tilde{h}_{t} \sigma_{n}^{2} \sigma_{x}^{4}}{w^{2} \overline{x_{t}^{2}}\left(\tilde{h}_{t}^{2} \sigma_{x}^{2}+\sigma_{n}^{2}\right)^{2}}=0$. Therefore $\delta h_{t}^{*}=0$ and the corresponding coefficients are given as the solution to the following optimization problem:

$$
\begin{aligned}
{\left[w_{3, t}, l_{3, t}\right]=} & \arg \min _{[w, l]}\left\{E\left[\left(x_{t}-w\left(\tilde{h}_{t} x_{t}+n_{t}\right)-l\right)^{2}\right]\right. \\
& \left.-\frac{\sigma_{n}^{2} \sigma_{x}^{2}}{\tilde{h}_{t}^{2}+\sigma_{n}^{2}}\right\}
\end{aligned}
$$

subject to

$$
\tilde{h}_{t}+\frac{l \overline{x_{t}}}{w \overline{x_{t}^{2}}}-\frac{1}{w}+\frac{\tilde{h}_{t} \sigma_{n}^{2} \sigma_{x}^{4}}{w^{2} \overline{x_{t}^{2}}\left(\tilde{h}_{t}^{2} \sigma_{x}^{2}+\sigma_{n}^{2}\right)^{2}}=0 .
$$

Hence, the proof follows.

\section{Simulations}

We provide numerical examples in different scenarios in order to illustrate the performances of the equalization methods. We first illustrate the performances of the channel equalization methods for a given perturbation bound. We demonstrate that the minimax equalization method yields the best worst case MSE performance among all methods for these simulations since it optimizes the worst case MSE with respect to the worst case channel coefficient. We next present the average MSE performance of each method over different channel perturbations. We show that the minimax regret method has better average MSE performance than the minimax and minimin equalization methods for these simulations.

In the first set of experiments, we randomly generate a transmitted signal $x_{t}$ of length 500 with mean 0.01 and variance 1 . We also generate a Gaussian channel noise $n_{t}$ with zero mean and unity variance. The channel estimates are constructed using $\tilde{h}_{t}=h_{t}+\delta h_{t}$, where $h_{t}=1.05$ and the perturbation $\delta h_{t}$ is randomly generated from a zero mean and $\epsilon$ standard deviation Gaussian distribution and truncated to give $\left|\delta h_{t}\right| \leqslant \epsilon$ with $\epsilon=0.03$ for each trial. Here, we label the method in Theorem 1 as "Minimax", the method in Theorem 2 as "Minimin", and finally the method in Theorem 3 as "Minimax regret". For each method and for each random perturbation, we find the corresponding equalizer parameters $w_{t}$ and $l_{t}$ to calculate the estimates of the transmitted signal $x_{t}$. After we calculate the mean-square errors for each method and for all random perturbations, we plot the corresponding sorted errors in ascending order in Fig. 2 for 200 trials. Since the minimax equalization method optimizes the worst case MSE with respect to worst possible perturbation, it yields the smallest worst case MSE among all methods for these simulations. However, the overall performance of the minimax method is significantly inferior to the minimax regret method due to its highly conservative nature. Furthermore, we notice that the minimax regret method provides better average performance compared to the minimax and the minimin methods and superior worst case performance compared to the minimin method for these simulations.

For the second experiment, we randomly generate 200 random perturbations $\delta h_{t}$, where $\left|\delta h_{t}\right| \leqslant \epsilon$ for different perturbation bounds and compute the averaged MSEs over 200 trials for the minimax, minimin and the minimax regret methods. In this case, we randomly generate a transmitted signal $x_{t}$ of length 500 with zero mean and variance 1 . The channel noise $n_{t}$ is generated from a Gaussian distribution with zero mean and unity variance. Here, we construct the estimates of the channel coefficient by $\tilde{h}_{t}=h_{t}+\delta h_{t}$, where $h_{t}=1.05$ and the perturbation $\delta h_{t}$ is randomly generated from a zero mean and $\epsilon$ standard deviation Gaussian distribution and truncated to give $\left|\delta h_{t}\right| \leqslant \epsilon$. In Fig. 3, we present the averaged MSEs for each method where the perturbation bound varies, 


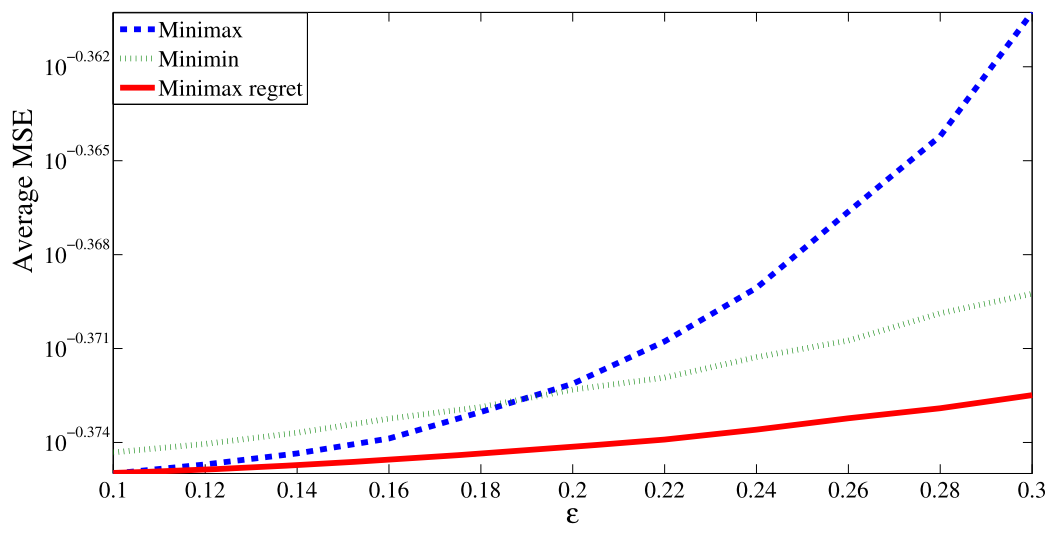

Fig. 3. Averaged MSEs for the minimax, minimin and minimax regret equalization methods over 200 trials when $\epsilon \in[0.1,0.3]$.

$\epsilon \in[0.1,0.3]$. We observe that the minimax regret method has the best average MSE performance over different perturbation bounds compared to the minimax and the minimin equalization methods.

\section{Conclusion}

In this paper, we investigated the channel equalization problem for time-varying flat fading channels when the channel coefficient is not accurately known. We analyzed three robust methods to equalize time-varying flat fading channels that incorporate the channel uncertainties into the problem formulation. We first studied the affine minimax channel equalization framework that optimizes equalizer parameters to minimize the worst case MSE in the uncertainty region. We next investigated the affine minimin channel equalization method, which minimizes the MSE for the most favorable channel coefficient in the perturbation bounds. Finally, we analyzed the affine minimax regret channel equalization framework, which minimizes the worst case regret in the uncertainty region. We explicitly provide the equalizer coefficients and the estimates of the desired signal for each method and for both zero mean and nonzero signals. We illustrated the performances of these equalization methods through simulations. We observed that the minimax approach leads to a better worst case MSE performances than the minimin and minimax regret approaches for these simulations. We also presented the average MSE performances of the equalization methods over different channel perturbations and showed that the minimax regret equalization method has the best average MSE performance among all methods for these simulations.

\section{References}

[1] J.G. Proakis, Digital Communications, McGraw-Hill Book Co., Singapore, 1995.

[2] L.M. Davis, S.V. Hanly, P. Tune, S.R. Bhaskaran, Channel estimation and user selection in the mimo broadcast channel, Digit. Signal Process. 21 (5) (2011) 608-618.

[3] M.U. Otaru, A. Zerguine, L. Cheded, Channel equalization using simplified least mean-fourth algorithm, Digit. Signal Process. 21 (3) (2011) 447-465.

[4] Y.C. Eldar, N. Merhav, A competitive minimax approach to robust estimation and random parameters, IEEE Trans. Signal Process. 52 (7) (2004) 1931-1946.

[5] Y.C. Eldar, A. Ben-Tal, A. Nemirovski, Minimax MSE-ratio estimation with signal covariance uncertainties, IEEE Trans. Signal Process. 53 (4) (2005) 1335-1347.

[6] M. Pilanci, O. Arikan, M.C. Pinar, Structured least squares problems and robust estimators, IEEE Trans. Signal Process. 58 (5) (2010) 2453-2465.

[7] S.S. Kozat, A.T. Erdogan, Competitive linear estimation under model uncertainties, IEEE Trans. Signal Process. 58 (4) (2010) 2388-2393.

[8] Y.C. Eldar, A. Ben-Tal, A. Nemirovski, Robust mean-squared error estimation in the presence of model uncertainties, IEEE Trans. Signal Process. 53 (1) (2005) 168-181.

[9] P.J. Huber, Robust estimation of a local parameter, Ann. Math. Statist. 35 (1964) 73-101.

[10] K.E. Schubert, A new look at robust estimation and identification, PhD dissertation, University of California, Santa Barbara, CA, 2003.
[11] N. Kalantarova, S.S. Kozat, A.T. Erdogan, Robust turbo equalization under channel uncertainties, IEEE Trans. Signal Process. 60 (1) (2012) 261-273.

[12] M. Tuchler, R. Koetter, A.C. Singer, Turbo equalization: Principles and new results, IEEE Trans. Commun. 50 (5) (2002) 754-767.

[13] S.S. Kozat, A.C. Singer, Universal switching linear least squares prediction, IEEE Trans. Signal Process. 56 (Jan. 2008) 189-204.

[14] M.C. Jeruchim, P. Balaban, K.S. Shanmugan, Simulation of Communication Systems, Kluwer Academic Publishers, 2002.

[15] D. Tse, P. Viswanath, Fundamentals of Wireless Communication, Cambridge University Press, 2005.

[16] T. Kailath, A.H. Sayed, B. Hassibi, Linear Estimation, Prentice-Hall, NJ, 2000.

[17] M. Tuchler, A.C. Singer, R. Koetter, Minimum mean squared error equalization using a priori information, IEEE Trans. Signal Process. 50 (3) (2012) 673-683.

[18] L.E. Ghaoi, H. Lebret, Robust solutions to least-squares problems with uncertain data, SIAM J. Matrix Anal. Appl. 18 (4) (1997) 1035-1064.

[19] S. Chandrasekaran, G.H. Golub, M. Gu, A.H. Sayed, Parameter estimation in the presence of bounded data uncertainties, SIAM J. Matrix Anal. Appl. 19 (1) (1998) 235-252.

[20] S. Chandrasekaran, G.H. Golub, M. Gu, A.H. Sayed, An efficient algorithm for a bounded errors-in-variables model, SIAM J. Matrix Anal. Appl. 20 (4) (1998) 839-859.

Mehmet Ali Donmez received the B.S. degrees from both the Department of Electrical and Electronics Engineering and the Department of Mathematics, Bogazici University, Turkey, with honors. He is currently an M.S. student at the Department of Electrical and Electronics Engineering of Koc University under the supervision of Professor Suleyman S. Kozat His current research interests are adaptive signal processing, intelligent systems, online learning, signal processing for communications, signal processing algorithms for mathematical finance and machine learning for signal processing.

Huseyin Atahan Inan received the B.S. degrees from the Department of Electrical and Electronics Engineering and the Department of Mathematics, Bogazici University, Turkey, with a 3.70 GPA. He is currently an M.S. student at the Department of Electrical and Electronics Engineering of Koc University under the supervision of Professor Alper Tunga Erdogan. His current research interests are adaptive signal processing, blind source separation and independent component analysis and signal processing for communications.

Suleyman Serdar Kozat received the B.S. degree with full scholarship and high honors from Bilkent University, Turkey. He received the M.S. and Ph.D. degrees in electrical and computer engineering from University of Illinois at Urbana Champaign, Urbana, IL, in 2001 and 2004, respectively.

After graduation, Dr. Kozat joined IBM Research, T.J. Watson Research Center, Yorktown, NY as a Research Staff Member in Pervasive Speech Technologies Group, where he focused on problems related to statistical signal processing and machine learning. While doing his Ph.D., he was also working as a Research Associate at Microsoft Research, Redmond, WA, in Cryptography and Anti-Piracy Group. He holds several patent applications for his works performed in IBM Research and Microsoft Research. After begin an Assistant Professor at Koc University for five years, Dr. Kozat is currently an Assistant Professor at the Electrical and Electronics Engi- 
neering Department, Bilkent University, Turkey. Dr. Kozat coauthored more than 50 papers in refereed high impact journals and conference proceedings and has several patent applications. Overall, his research interests include intelligent systems, adaptive filtering for smart data analytics, online learning and machine learning algorithms for signal processing.

Dr. Kozat has been serving as an Associate Editor for the IEEE Transactions on Signal Processing and he is a Senior Member of the IEEE. He has been awarded IBM Faculty Award by IBM Research in 2011, Outstanding Faculty Award by Koc University in 2011, Outstanding Young Researcher Award by the Turkish National Academy of Sciences in 2010, ODTU Professor Dr. Mustafa N. Parlar Research Encouragement Award in 2011 and holds Career Award by the Scientific Research Council of Turkey, 2009. Dr. Kozat has won several scholarships and medals in international and national science and math competitions. 